\title{
Reducing structural-element salience on a source problem produces later success in analogical transfer: What role does source difficulty play?
}

\author{
ANDRÉ DIDIERJEAN \\ University of Provence and CNRS, Aix-en-Provence, France \\ and \\ SANDRA NOGRY \\ Claude Bernard University, Lyon, France
}

\begin{abstract}
Two experiments in reasoning by analogy were conducted to study the role of inducing source difficulty by reducing the salience of the source's structural elements. Three nonexclusive hypotheses were tested. According to the first, a difficult source problem improves analogical transfer because it increases the probability that the subject will notice the similarity between the source and the target. For example, errors made on both the source and the target can enhance the subject's awareness of the similarity between the two problems. According to the second hypothesis, a source that is difficult to solve is memorized better than an easier source. According to the third, source-problem difficulty affects the degree of abstractness in the representation of the solution elaborated by subjects. Experiment 1 showed that the higher frequency of spontaneous transfer between the source and the target when the source problem was difficult (Gick \& McGarry, 1992) could be replicated in a cued-transfer situation. Experiment 2 showed that subjects given a difficult source, one in which the important element was not very salient, were better at categorizing isomorphic problems on the basis of structural features than were subjects given an easy source. The discussion deals with the implications of these results for the hypotheses tested and, more generally, for reasoning by analogy and education in general.
\end{abstract}

To learn a concept, is it preferable to study an example in which the important elements stand out or one that is more difficult because the important elements are less conspicuous? Deliberately making the problem more difficult leads subjects to make more errors during the solving process, but do such errors have a positive impact on learning? Some authors have argued that obstacles and errors impinge upon the learning process, because subjects remember past solving sequences whether the sequences are good or bad (Paas \& Van Merriënboer, 1994), but many others have claimed that obstacles and errors enhance recall (Patalano \& Seifert, 1994), learning (VanLehn, 1998), and analogical transfer (Gick \& McGarry, 1992). The present study addresses this last point - that is, the impact of difficulties encountered by subjects during source-problem solving on the subsequent solving of analogical problems.

\section{Reasoning by Analogy}

One way of solving a problem is to retrieve and adapt an already solved problem whose solution seems iso-

This work was partially supported by a grant from the CNRS, programme STIC-SHS "société de l'information." We thank Agnès Grimmaud for her help in data collection, as well Mary Gick and an anonymous reviewer for insightful comments on previous drafts of this article. Correspondence should be directed to A. Didierjean, LPC-CNRS et Université de Provence, 29, avenue Robert Schuman, 13621 Aix-enProvence, France (e-mail: andre@up.univ-mrs.fr). morphic. In cognitive psychology, a large number of studies have attempted to examine and model the mechanisms at play in reasoning by analogy (Falkenhainer, Forbus, \& Gentner, 1989; Holyoak, 1984; Holyoak \& Thagard, 1989; Hummel \& Holyoak, 1997; Keane, Ledgeway, \& Duff, 1994). In the particular case of problem solving, the classical distinction is made between two forms of analogical reasoning: cued and spontaneous transfer (Gick \& Holyoak, 1980, 1983). In their original studies, Gick and Holyoak $(1980,1983)$ showed that when given two isomorphic problems in succession without being informed of the similarity between them, few subjects transfer the first problem solution to the second (this situation is called "spontaneous" transfer). However, in a cued-transfer situation, in which subjects are informed of the connection between the first and second problems, source-to-target transfer is much more frequent. This result has been replicated in many studies since then (e.g., Bassok, 1990; Beveridge \& Parkins, 1987; Catrambone \& Holyoak, 1989; Keane, 1985, 1987; Novick \& Holyoak, 1991; Reed, Dempster, \& Ettinger, 1985). Further studies in this line have shown that the frequency of spontaneous source-to-target transfer could be boosted appreciably by increasing the degree of surface similarity between the two problems (Holyoak \& Koh, 1987) - the more surface features the source has in common with the target, the higher the spontaneous trans- 
fer rate. Research on reasoning by analogy has often dealt with the potential role of surface features. Such features apparently affect analogical transfer through the impacts they have on source retrieval (Holyoak \& Koh, 1987), source-to-target mapping (Heydenbluth \& Hesse, 1996; Ross, 1987, 1989), and even target encoding (Ross \& Bradshaw, 1994).

\section{Role of Source Difficulty in \\ Reasoning by Analogy}

While the role of surface features has been examined in numerous studies, Gick and McGarry (1992) showed that a second factor also seems to be critical in analogical transfer: the salience of the source's structural features. These authors conducted a study in which the subjects had to solve the so-called mutilated checkerboard problem (presented in Figure 1), a difficult puzzle that few of the subjects managed to solve (Kaplan \& Simon, 1990). In this problem, the subjects have to determine whether a checkerboard with two of its opposing corner squares removed can be covered by a set of dominoes, each of which covers exactly two squares. To correctly solve the problem, the idea of parity must be used: A given domino always covers two adjacent squares, one black and one white,

You are given a checkerboard and 32 dominoes. Each domino covers exactly two adjacent squares on the board. Thus, the 32 dominoes can cover all 64 squares of the checkerboard. Now suppose two squares are cut off at diagonally opposite corners of the board. If possible, show how you would place 31 dominoes on the board so that all of the 62 remaining squares are covered. If you think it is impossible, give a proof of why.

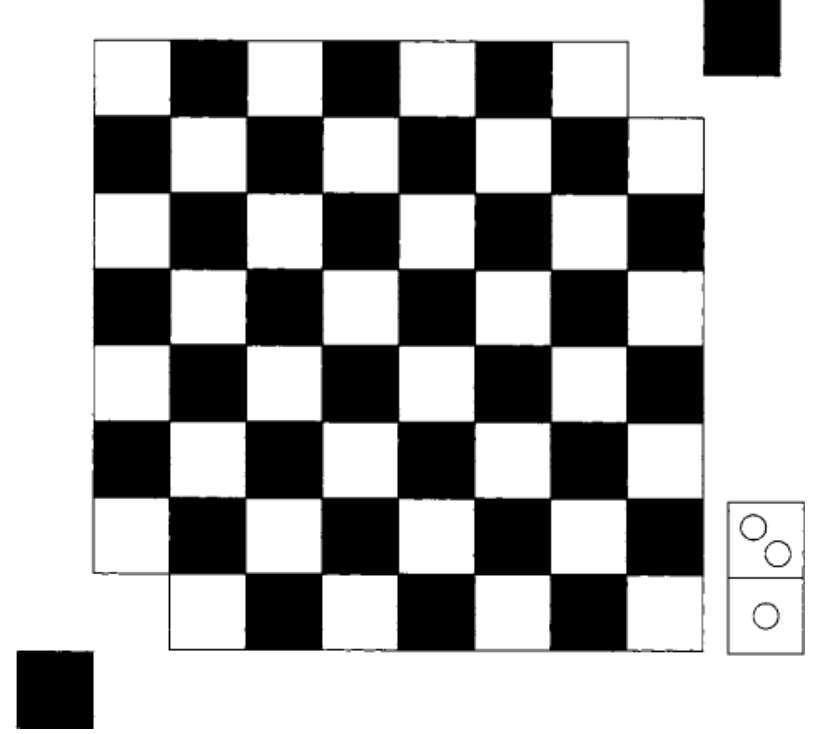

Figure 1. The standard mutilated checkerboard problem. From "Learning from mistakes: Inducing analogous solution failures to a source problem produces later successes in analogical transfer," by M. L. Gick and S. J. McGarry, 1992, Journal of Experimental Psychology: Learning, Memory, \& Cognition, 18, p. 625. Copyright 1992 by the American Psychological Association. Reprinted with permission. yet two black squares have been removed. Even though the total number of squares matches the number of domino halves, the problem is impossible, because no matter how the dominoes are laid out, there will always be one domino and two white squares left in the end.

First, Gick and McGarry (1992, Experiment 1) showed, as could be expected, that when researchers presented an isomorphic problem that all subjects were able to solve (the "partner problem," given in Appendix A) before the checkerboard problem, there was no spontaneous transfer of the first problem to the second (see also Gick \& Holyoak, 1980, 1983). Later (Gick \& McGarry, 1992, Experiments 2 and 3 ) they showed that the degree of complexity of the source played a determining role in the frequency of spontaneous transfer. The subjects in these experiments were given a source problem called "the dinner party" (isomorphic to the checkerboard problem). Half of the subjects were given an "easy" version of the source in which the notion of parity was made plain (see Figure 2), the other half were given a more "difficult" version where the notion of parity was less apparent. ${ }^{1}$ The only difference between the two versions was the greater or lesser salience of the structural element (parity) that had to be taken into account to solve the problem correctly; in the easy version, this element was more conspicuous than in the difficult version. Note that neither version was closer to the target in terms of surface features.

The results showed that the subjects given the difficult source version did better on the target checkerboard problem than did the subjects given the easy version. Thus, while surface features seem to play a role in spontaneous transfer, reducing the salience of the important structural element in the source also seems to be a determining factor. Three nonexclusive hypotheses can potentially account for this finding.

In the first hypothesis, since the target is difficult, the fact of also making the source difficult promotes sourceto-target transfer because subjects are more likely to notice the source-target link in this case. A difficult source should therefore be favorable because subjects make mistakes, and the mistakes made when solving the source and target help subjects see the connection between the two problems. When subjects make a mistake on the target that is similar to one made on the source, they become aware of the existence of the source-target similarity. As Gick and McGarry (1992, p. 635) stated, "For example, if subjects are attempting covering solutions that they think should work in the checkerboard target problem, but all solutions seem to fail, they may notice a similarity to failures that occurred in the source problem (e.g., two squares are always left over)." In this hypothesis, errors, like surface features, act as cues and take the subjects from a spontaneous-transfer to a cuedtransfer situation. Thus, by making a source more difficult, one increases the probability that the subjects will notice the utility of using the source to solve the target.

In the second hypothesis, reducing the salience of the important structural element improves the transfer because subjects memorize the source and its solution better when 
Thirty-six people, 18 men and 18 women, are at a dinner party, as illustrated schematically below. The 36 people are seated at 18 tables. Each table only seats two people, a man and a woman, who are sitting right next to each other, either in a horizontal or vertical direction. If two women leave, as illustrated by the arrows in the diagram below, can 17 tables be arranged to seat the other 34 people? The people are not allowed to move. Explain your reasoning.

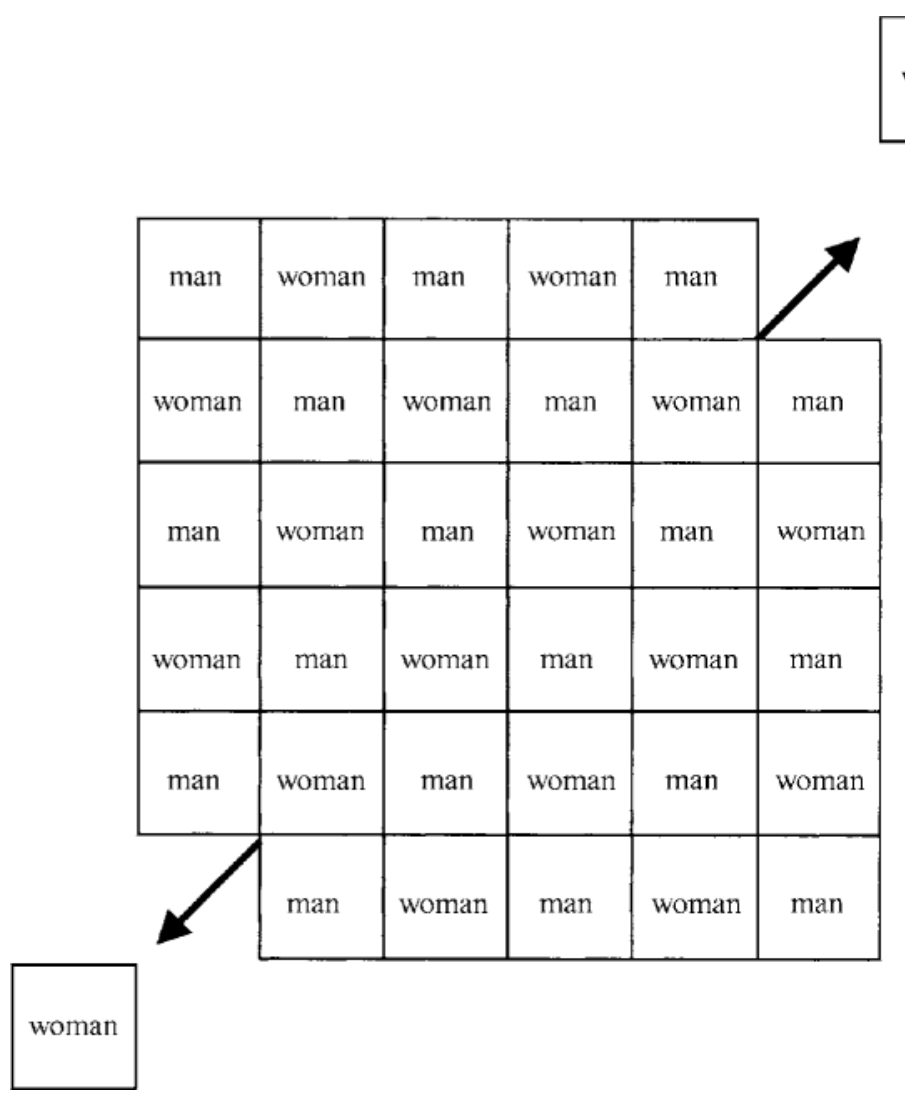

Figure 2. Easy version of the dinner party problem. From "Learning From Mistakes: Inducing Analogous Solution Failures to a Source Problem Produces Later Successes in Analogical Transfer," by M. L. Gick and S. J. McGarry, 1992, Journal of Experimental Psychology: Learning, Memory, \& Cognition, 18, p. 630. Copyright 1992 by the American Psychological Association. Reprinted with permission.

they have trouble solving it. In accordance with this theory, Patalano and Seifert (1994) showed that subjects were better at remembering problems they had solved erroneously than problems they had solved correctly.

In the third hypothesis, good memorization of the source may not suffice for solving the target problem. Since Gick and Holyoak's (1983) work, many studies have shown that analogical reasoning, particularly when it is interdomain, often requires building knowledge that is not dependent upon surface features. This kind of abstract knowledge, traditionally called a schema, can be built from several sources via a source comparison process (Cummins, 1992; Gick \& Holyoak, 1983; Gick \& Paterson, 1992), can take place as the source problem is being mapped to the target (Didierjean, 2003; Gentner,
1989; Holyoak \& Thagard, 1989; Ross \& Kennedy, 1990), or can be derived from the analysis of a single source problem (Ahn, Brewer, \& Mooney, 1992; Elio \& Anderson, 1981; Kieras \& Bovair, 1986). In the latter case, the subject's self-explanations appear to be the critical factor (Catrambone, 1995, 1996; DeJong \& Mooney, 1986; Mooney, 1990). To illustrate, in Gick and McGarry's Experiment 1 , where subjects were given the partner problem as the source (see Appendix A), one can assume that no matter how well they memorized the source, they did not solve the target because they failed to extract the concept of parity from the surface features of the man/ woman couple and to realize its importance for solving the problem. According to the third hypothesis, reducing the salience of the important structural element in the 
source leads subjects to build a representation of the solution that encodes the key element in a more abstract fashion. This experimental manipulation could lead the subjects' attention to the role of parity in the solution, and thus to more generalization as a result of a self-explanation process (Catrambone, 1995, 1996; DeJong \& Mooney, 1986; Mooney, 1990). More specifically, by thinking about the role of the structural element they did not initially take into account, subjects may construct a representation of the source in which the component "two women are missing" is replaced by a more abstract component, such as "two like elements are missing." (For this type of generalization phenomenon, see Sander \& Richard, 1997; Schank, 1982.)

\section{Overview of the Experiments}

The aim of this study, which used Gick and McGarry's (1992) problems, was to find out why solving a particular source problem in which the important structural element is not salient facilitates reasoning by analogy more than does solving an easy source problem in which that element stands out. In Experiment 1, we looked at whether the effect of source-problem difficulty in analogical transfer persists in a cued-transfer situation. Our prediction, as suggested in Hypothesis 1 above, was that if working on a difficult source facilitates analogical reasoning solely because it helps subjects notice the sourcetarget similarity (for example, because they make the same mistakes on the two problems), then the difference between the subjects given a difficult source and others given an easy source should disappear as soon as the subjects are no longer in a spontaneous-transfer situation. If, on the other hand, reducing the salience of the important structural element reinforces memorization (Hypothesis 2) or promotes the building of knowledge that incorporates parity in an abstract way (Hypothesis 3 ), then the difference should still exist in cued transfer. Experiment 2 specifically tested the hypothesis that source difficulty plays a role in knowledge generalization. After working on one of the sources used in the previous experiment, the subjects were asked to perform a categorization task. They had to sort different versions of the checkerboard problem on the basis of whether the problem seemed structurally like or unlike the dinner party problem. Our prediction here was that if the subjects in a difficult-source group build knowledge that incorporates in an abstract way the importance of parity in the problem solution (e.g., by integrating the generalization that two like elements, not simply two women, are removed), then they should be better at categorizing problems on the basis of this structural feature than are the subjects in the other group (Chi, Feltovich, \& Glaser, 1981; Cummins, 1992).

\section{EXPERIMENT 1}

Gick and McGarry (1992, Experiment 2) showed that the subjects given the easy dinner party problem as the source had a lower spontaneous transfer rate than others given the difficult version. If reducing the salience of the important structural element is critical because it helps the subjects realize the utility of the source for solving the target, then the difference between the two groups should disappear when the subjects are informed of the connection between the two problems. On the other hand, if reducing the salience of the important element improves memorization of the source or leads the subjects to build a representation of the solution that encodes the role of parity in a more abstract way, the difference between the two groups should persist.

Note that in Gick and McGarry's (1992) study, the subjects who did not spontaneously transfer the source to the target underwent a second phase in which they were informed of the similarity between the two problems and then tried to solve the target problem again. Success on the target increased to the same extent in the two groups. Since this phase came after the spontaneous-transfer phase and only concerned the subjects who had failed the first time, however, it is difficult to draw any conclusions about this aspect of Gick and McGarry's results. In our first experiment, therefore, the subjects solved the dinner party problem (easy or difficult version), read the solution, and were then told outright that the solution to that source problem could help to solve the target problem as well. Only then did they attempt a solution to the checkerboard problem.

Another aim of this experiment was to study the role of errors in source-to-target transfer. Gick and McGarry demonstrated the role of a particular type of source error: failure to take parity into account. According to these authors, if a difficult source facilitates spontaneous transfer to the target, this occurs partly because subjects make the same errors on the two problems, and these shared errors help them notice the utility of the source in solving the target. The present study thus analyzes the errors made on the source and relates these errors to eventual successful solution of the target.

\section{Method}

Subjects. The subjects were 90 first- and second-year psychology students at the University of Provence in Aix-en-Provence, France. Their mean age was 20 years 4 months ( $S D=14$ months). None of the subjects had heard of the mutilated checkerboard problem before the experiment.

Materials. Three different problems were used: a French version of the checkerboard problem (see Figure 1) and two French versions of Gick and McGarry's dinner party problem. The easy version of the dinner party problem (Gick and McGarry's "verbal-plus-diagram" version) is presented in Figure 2 (see Appendix B for the difficult version). In the difficult version of the problem ("diagram-only"), the words two women leave were replaced by two people leave and the phrase a man and a woman was omitted. Thus, in comparison with the easy version, the difficult version was "difficult" solely because it contained the information needed to solve the parity problem only in the diagram.

Procedure. The subjects were tested two at a time, but each one worked alone. The subjects were informed that they would have to do some other problems later and were randomly assigned to one of two conditions: easy or difficult source. They then received a pen and the 
first problem and were asked to read the problem statement carefully, to use the diagram as an aid for solving the problem, and to feel free to jot things down on the sheet of paper provided. If they found the solution, they were told to write it down on the answer sheet. The subjects were allotted $5 \mathrm{~min}$ for solving the source problem. When the time was up, the subjects were given a sheet of paper showing the solution (see Appendix C) and were asked to read it carefully. When they felt they understood the solution well, they were asked to write it down again, in their own words, on the back of the sheet provided.

After this phase, the subjects in both groups had to solve the checkerboard problem. When handing out the problem, the experimenter repeated the instructions given for the source problem and then attracted the subjects' attention to an important point by saying, "This problem is very much like the one you just solved. While you're solving it, try to remember the solution you just saw, since it will help you solve this new problem." Five min were allotted once again. Afterward, the experimenter told the subjects to recall and write down the source-problem solution and then asked them whether they had ever seen either problem before the experiment.

\section{Results}

For both experiments, the subjects' source and target solutions were scored blindly by two judges who were unaware of the experimental hypotheses. In Experiment 1, the disagreement rate between the judges was $8 \%$. After discussing the scoring criteria, they reached an agreement on all problems. In Experiment 2 (in which only the sources were scored), the disagreement rate between the judges was $0 \%$.

Source-problem performance. The subjects' dinner party solutions were scored using Gick and McGarry's (1992) criteria. An answer was considered correct if it contained at least two of the following elements: (1) a table seats one man and one woman; (2) two women have left; (3) two men remain who cannot be seated at a table.

The results obtained for the source problem showed, as expected, that the subjects given the difficult version of the dinner party problem produced fewer correct parity solutions than did subjects given the easy version $(4.5 \%$ vs. $28.9 \%)\left[\chi^{2}(1, N=90)=9.68, p<.01\right]$.

The recalled solutions produced immediately after source reading were also scored. The difference between the two groups was nonsignificant $(88.8 \%$ for the difficult version vs. $95.5 \%$ for the easy version) $\left[\chi^{2}(1, N=\right.$ 90) $=1.40, p=.3]$.

Target-problem performance. The cued-transfer rate was higher for the group given the difficult version of the dinner party problem as the source than it was for the group given the easy version $(40 \%$ vs. $17.8 \%)\left[\chi^{2}(1\right.$, $N=90)=5.41, p<.05]$.

We then related source success or failure to target success. For the two groups pooled, very few subjects succeeded on the source problem (15 subjects out of 90,13 in the easy-source and 2 in the difficult-source group). Among these subjects, only 5 correctly solved the target problem.

Relationship between source-problem errors and target-problem success. The errors were classified into three categories, as proposed by Gick and McGarry (1992): nonparity solution failures, one-feature solution failures, and other solution failures. Nonparity solution failures did not mention parity features and showed clear evidence of a problem representation that was not based on parity. One-feature solution failures mentioned only one parity feature. Other incorrect solutions included those stating that the solution was possible (e.g., by violating problem constraints), blank answer sheets (the majority), and solutions that were unclear.

The response to the source and target problems for each group are cross-tabulated by type in Appendix D. The main finding here, as in the Gick and McGarry (1992) study, was that subjects given a difficult source produced more nonparity solution failures on the source than did subjects given an easy source $(31 \%$ vs. $9 \%)\left[\chi^{2}(1, N=\right.$ $90)=6.94, p<.01]$. However, target success was unrelated to the type of source error. For all groups pooled, only $28 \%$ of the subjects who produced nonparity solution failures on the source problem succeeded on the target, versus $29 \%$ target success for the other response types $\left[\chi^{2}(1, N=90)=.01, p=.91\right]$. Note that while nonparity errors were not overall predictors of success, they did not have the same status in the two groups. In the easy-source group, none of the subjects who made nonparity errors on the source managed to solve the target. In the difficult-source group, however, a third of the subjects who made such errors went on to solve the target correctly. This finding confirms the fact that while this type of error does not predict success, the experimental manipulation performed on the two groups did have an impact on whether the subjects grasped the concept of parity.

Source-problem recall. The recall of the sourceproblem solution required after solving the target was scored on a pass-fail basis by two separate judges, using the same criteria as above. The two groups did not differ significantly $\left[\chi^{2}(1, N=90)=.56, p=.45\right]$.

\section{Discussion}

The first important finding of this experiment is that although the subjects did have less trouble with the easy version of the source problem (in terms of success or failure) than with the difficult version, both versions in fact turned out to be difficult. Very few subjects correctly solved the problem, and therefore the observed difference on the target problem between the two difficulty groups cannot by ascribed solely to a difference in source success. Concerning the errors made, Gick and McGarry (1992, p. 631) stated, "A crucial prediction was that subjects would produce more nonparity solution failures to the diagram-only version of the dinner party problem than to the verbal versions." Our results, like Gick and McGarry's, confirm this prediction and thus show that the difference generated by the two versions of the source problem did indeed affect the subjects' understanding of the key concept in the solution: parity. Note, however, that having trouble with parity on the source was not enough to ensure success on the target. Although we found that the subjects produced more nonparity solution failures when they had worked on a difficult source, the dif- 
ference observed on the source did not account for the difference observed on the target.

Our main finding was that this experiment replicated Gick and McGarry's (1992) results for cued transfer. This finding clearly argues for the claim that the observed difference between the two experimental groups was not due solely to the fact that complicating the sourcesolving process allowed subjects to become aware of the utility of the source in solving the target. Experiment 2 was aimed at testing our other two hypotheses by examining the degree of generality of subjects' knowledge after working on the source.

\section{EXPERIMENT 2}

The results of Experiment 1 allow us to suggest that if the frequency of spontaneous transfer is greater when subjects study a source that does not feature prominently the critical element for solving the problem, this effect does not occur solely because errors induced by the difficult version increase awareness of source-target similarity. Two possible accounts could explain these results: One possibility is that subjects memorized the source and its solution better when they had trouble solving it. Patalano and Seifert (1994), for instance, showed that subjects were better at remembering problems they had solved erroneously than others they had solved correctly. Note, however, that in our Experiment 1, the two groups did not differ on the source recall task that came after target-problem solving. The other possibility is that the subjects who had trouble with parity on the source built a representation in which this concept was incorporated in a more abstract way (e.g., Needham \& Begg, 1991). Note that while each of these hypotheses alone can account for the results, they are not exclusive: Generalization can sometimes be accompanied by good memorization of surface features. (Reeves \& Weisberg, 1994, speak of "conservative" generalization in this case; see also Marmèche \& Didierjean, 2001).

In this second experiment, the generality of subjects' representations of the role of parity after studying the source was tested using a categorization task. After trying to solve the dinner party problem (in either its easy or difficult version), the subjects had to sort variations of the mutilated checkerboard problem on the basis of proximity to the dinner party problem. The extent to which variations of the checkerboard problem were close to the dinner party problem depended on surface features (whether or not the removed squares were in the corner) and structural features (whether or not the removed squares were the same color). Figure 3 presents an example of the different checkerboard variations given to the subjects.

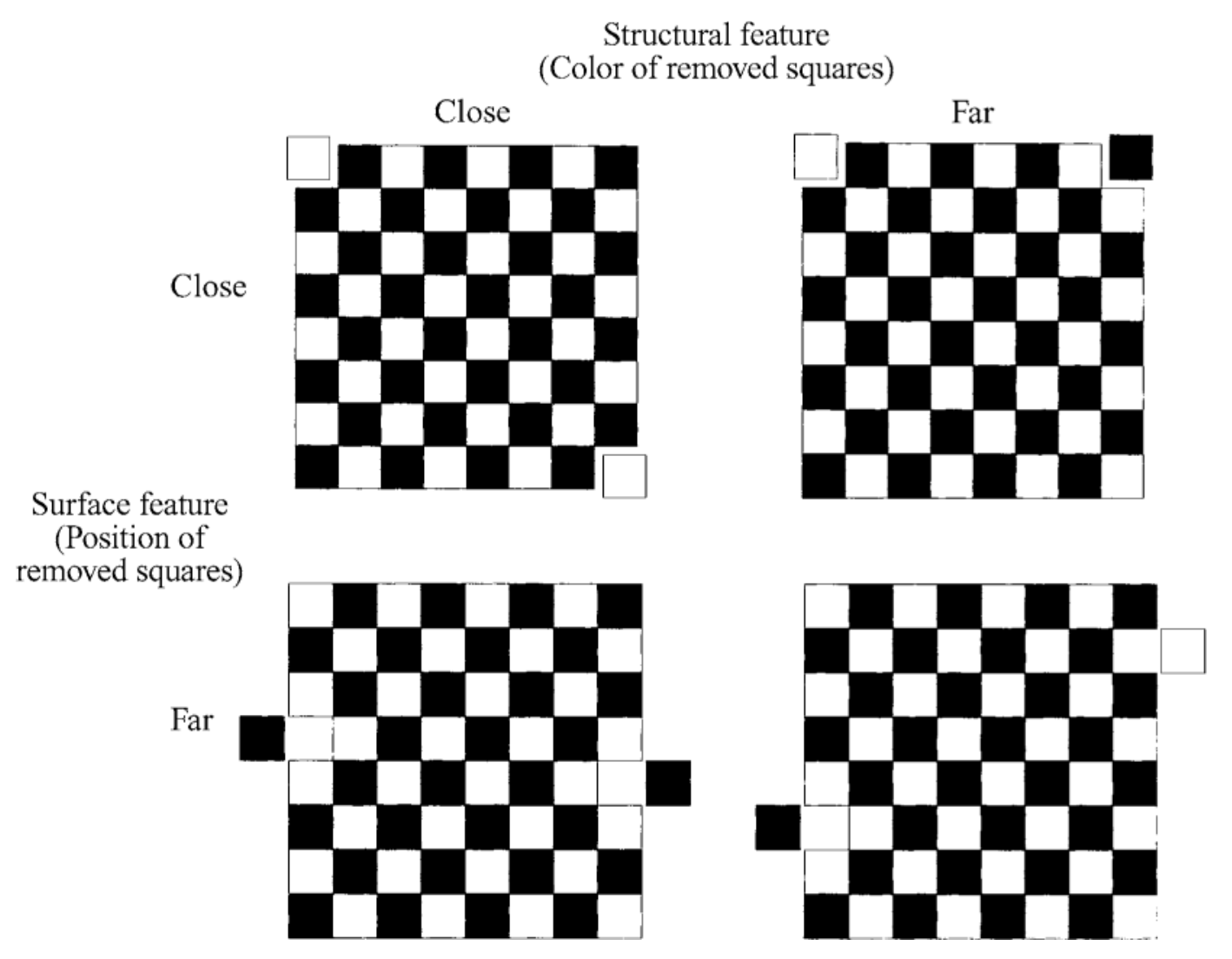

Figure 3. Example of checkerboards used for the categorization task. 
If a difficult source has an impact on transfer because it causes the construction of knowledge in which the notion of parity is represented abstractly, then the subjects who worked on the difficult version of the dinner party problem should be better at sorting problems according to this structural feature (two same-color squares removed) than the subjects who worked on the easy version would be. If a difficult source has an impact on transfer only because it promotes source memorization, performance should be equivalent in the two groups. Indeed, no matter how well the source is memorized, one cannot separate problems with two same-color squares removed from others with different-color squares removed unless one understands that parity is a key element in this type of problem.

\section{Method}

Subjects. The subjects were 80 first- and second-year psychology students at Lumière University in Lyon, France. Their mean age was 22 years 3 months ( $S D=60$ months). Six of the subjects had already heard of the mutilated checkerboard problem ( 2 in the easy and 4 in the difficult condition), so their responses were not included in the data analyses.

Materials. For the source-problem solving phase,we used the same materials that had been used in Experiment 1. For the test phase, eight problems, the original problem and seven variations, were generated from the French version of the mutilated checkerboard problem. The variations were constructed by taking out sameor different-color squares and by changing the location of the removed squares (from either other corners or around the edge).

Procedure. The subjects were tested in groups of 15 , but each worked alone. The subjects were randomly assigned to one of two conditions: easy or difficult source. They were then given a pen and the first problem and asked to read the problem statement carefully, to use the diagram as an aid for solving the problem, and to feel free to jot things down on the sheet provided. Each was allotted $5 \mathrm{~min}$ for solving the source problem. If the subjects found the solution, they were asked to write it down on the answer sheet.

When the 5 min were up, each subject received a sheet of paper showing the solution to the source problem (see Appendix C) and was asked to read it carefully. When subjects felt they understood the solution well, they were told to write it down again, in their own words, on the back of the sheet provided.

After this phase, the subjects in both groups had to sort the eight versions of the mutilated checkerboard problem into two piles, one for versions they thought could be solved like the source problem and the other for those that seemed very different. A time limit of 5 min was allotted for reading the problem statements and sorting the problems.

Afterward, the experimenter asked the subjects if they had heard of either problem before the experiment.

\section{Results and Discussion}

Source-problem performance. As in the first experiment, the solutions the subjects proposed to the dinner party problem were scored using Gick and McGarry's (1992) criteria. The source-problem results showed that the subjects who had the difficult version of the problem found the correct solution less often than the subjects who had the easy version $(5.5 \%$ vs. $21.6 \%)\left[\chi^{2}(1, N=\right.$ 74) $=3.799, p=.051]$.

The recalled solutions were also scored. The difference between the two groups was nonsignificant (94.4\% for the difficult version vs. $97.4 \%$ for the easy version) $\left[\chi^{2}(1, N=74)=0.406, p=.524\right]$.

Problem categorization. The problem categorizations proposed by the subjects were scored on a pass-fail basis. A correct sorting was one in which all four problems with similar structural features were put in the same pile; all other responses were considered incorrect.

The percentage of correct sortings was significantly higher for the group who had as their source the difficult version than it was for those who had the easy version $(55.5 \%$ vs. $24.3 \%)\left[\chi^{2}(1, N=74)=7.880, p<.01\right]$.

Sorting-task success was not linked to whether the source problem was solved correctly or incorrectly (all conditions pooled: $44.4 \%$ vs. $38.5 \%$, respectively) $\left[\chi^{2}(1\right.$, $N=74)=0.003, p=.954]$. Looking solely at the sorting results for the subjects who failed on the source problem, we obtained a similar result as when all subjects were pooled: The difficult-source subjects were much better at categorizing the problems on the basis of their structural features $(55.9 \%$ vs. $20 \%$ success $)\left[\chi^{2}(1, N=\right.$ 64) $=8.621, p<.01]$.

\section{GENERAL DISCUSSION}

The goal of these experiments was to examine the role of source-problem difficulty in reasoning by analogy (Gick \& McGarry, 1992). Three nonexclusive hypotheses were set forth: First, reducing the salience of the critical element for solving the problem affects spontaneous transfer because it helps subjects see the analogy between the two problems. Second, source memorization among subjects is better with the difficult version. Third, reducing the salience of the important solving element affects spontaneous transfer because reduced salience induces subjects to build a representation of the source that encodes that element in an abstract way-for example, by including the constraint "two like elements are missing" rather than "two women are missing" in the representation of the solution. Experiment 1 replicated Gick and McGarry's results in a cued-transfer situation. Experiment 2 showed that a difficult source enhanced subjects' performance on a sorting task: The subjects who had tried to solve the difficult version of the source were better at categorizing isomorphic problems on the basis of the structural feature (parity) that had not been highlighted in the source problem.

These results clearly rule out the first hypothesis as an exclusive hypothesis: The role of source-problem difficulty cannot be ascribed solely to its impact on awareness of source-target similarity. They also allow us to rule out Hypothesis 2 (better source memorization), which by itself cannot account for the results of Experiment 2: If the subjects more often relied on structural than on surface similarity to sort the problems, they did so because the source-problem representations that they built incorporated the manipulated structural feature in a more abstract way. In addition, neither difficulty group performed better than the other in the source recall phase 
run after the target problem in Experiment 1, so we can eliminate Hypothesis 2 even as a complementary account. The latter result is similar to Gick and McGarry's 1992 finding with the same material; see also Novick and Holyoak, 1991. Only Hypothesis 3, that difficulty induces subjects to build a more abstract representation of the solution, explains the finding that reducing the salience of the structural element of the source improves transfer to the target.

Two questions remain to be clarified. First, do the errors triggered by the difficult source play a role beyond that demonstrated in our experiments - that is, beyond facilitating more abstract encoding of the important structural element? According to Gick and McGarry (1992), when faced with a difficult source, "subjects incorporate the failed solution into the representation of the problem, as an additional constraint" (p. 624). In support of this argument, Gick and McGarry showed (Experiment 4) that copying problem solutions containing mistakes was a better source of later transfer than was copying error-free solutions. However, it remains to be shown experimentally that the source representations in this case did in fact contain the failed solutions.

Second, although our results show that source difficulty plays a role in the abstraction level of the source representation, precisely what that role is remains unclear. One hypothesis is that making a source difficult by reducing the salience of the important structural element leads subjects to think about the role of this element, and thus to generalize more, by way of self-explanation. Many studies have shown that small changes in a problem solution may or may not trigger generalization, and that when they do, it is because they lead subjects to ask themselves questions about the role that changes play in the solution. Catrambone $(1995,1996)$ showed that when labels were added to a problem solution, subjects generalized, even when the labels were uninformative. The author explained that this finding resulted because the added labels led subjects to wonder about the reasons for the labels and their locations, and thus triggered a selfexplanation process. In a similar manner, reducing the salience of parity in the source could attract subjects' attention to the role of parity in the solution. The difficultsource subjects studying the solution to the dinner party problem may therefore construct a representation in which the key to solving the problem is that "two like elements" are withdrawn, not "two women."

We cannot altogether rule out the possibility that the generalization observed here did not take place when the source was being processed, but when the target problem was being solved (Experiment 1) or categorized (Experiment 2). This type of generalization (occurring during the transfer of a source to a target) has been observed in various domains (e.g., for problem solving, see Ross $\&$ Kennedy, 1990; for a discussion of this type of phenomenon in artificial grammar tasks used to study implicit learning, see Wright \& Whittlesea, 1998). In our study, however, this hypothesis does not seem compati- ble with the results of Experiment 2. Indeed, under this theory, how could one explain the observed difference between the two groups on the target knowing that their source memorization was equivalent?

\section{Implications of Our Results in the Field of Reasoning by Analogy}

We argue here that source difficulty in reasoning by analogy mainly plays a role because it changes the nature of the knowledge elaborated about the source problem. In the literature on analogical reasoning, three phases are generally distinguished (see, e.g., Anolli, Antonietti, Crisafulli, \& Cantoia, 2001; Gick \& Holyoak, 1983; Holyoak, 1984; Keane, 1987; Reeves \& Weisberg, 1994; Ross, 1987, 1989): (1) target and source encoding (Ross \& Bradshaw, 1994); (2) retrieval (Holyoak \& Koh, 1987; Keane, 1985, 1987; Novick \& Holyoak, 1991), selection (Clement \& Gentner, 1991; Gentner \& Toupin, 1986; Holyoak \& Koh, 1987; Holyoak \& Thagard, 1989), or access (Ross, 1987; Ross \& Kennedy, 1990; Schunn \& Dunbar, 1996); note that within the access phase, Anolli et al. also suggest differentiating between two processes: becoming aware of the existence of a similarity between source and target and identifying source-target connections; and (3) mapping (Clement \& Gentner, 1991; Gentner \& Toupin, 1986; Holyoak \& Koh, 1987; Holyoak \& Thagard, 1989), use (Schunn \& Dunbar, 1996), applying (Gick \& Holyoak, 1983; Gick \& McGarry, 1992; Ross, 1987, 1989), or adapting (Keane, 1996; Novick \& Holyoak, 1991).

Our work suggests that in certain studies, Phases 2 and 3 of reasoning by analogy cannot be examined without looking into the nature of the knowledge built from the source. Models of reasoning by analogy have mainly focused on the retrieval and mapping phases (Falkenhainer et al., 1989; Holyoak, 1984; Holyoak \& Thagard, 1989; Hummel \& Holyoak, 1997; Keane, Ledgeway, \& Duff, 1994), but most of the time, the source has had a representation format that was defined in advance by the authors of the model. To expand these models, it would undoubtedly be useful if they included whether the knowledge elaborated by the reasoner is abstract (Cummins, 1992; Gick \& Holyoak, 1983) or specific (Reed, 1987, 1989; Reed \& Bolstad, 1991; Ross, 1984, 1987, 1989), as well as which processes are implemented to use that knowledge and what conditions trigger them. Varying the difficulty of the source seems to be a good means of acting upon knowledge generalization processes.

\section{CONCLUSION}

The present results replicated Gick and McGarry's (1992) findings and showed that working on a "difficult" problem (one in which the key element of the solution is not brought to the subject's attention) is more likely to facilitate source-to-target transfer than is working on an "easy" problem. The results also showed that presenting examples in this way can have a genuine effect on the 
learning of the targeted concept: The degree of abstraction of the concept is affected. This nonintuitive finding goes against common teaching practices. In most cases, when students have to learn a new theoretical concept, the teacher deliberately highlights the concept in the examples presented. In the research on learning by examples, a large number of studies have shown that the examples given to learners may or may not trigger a generalization process, depending on how they are presented. Subjects generalize more if the examples studied are not too redundant (e.g., Chandler \& Sweller, 1991), if diagrams are integrated into the problem statement rather than given separately (Chandler \& Sweller, 1991, 1992, 1996; Sweller, Chandler, Tierney, \& Cooper, 1990), and if examples are structured by titles that separate the steps in the solving process (Catrambone, 1995, 1996). However, as Sweller $(1988,1994,1999)$ argued in his cognitive load theory, these surface modifications are always aimed at lightening the load in working memory, which is likely to facilitate the generalization process. However, in our study - which certainly needs to be reproduced for other problems, and particularly for types other than insight problems - making the source more complicated in no way lightened the working memory load, but it nevertheless led to more generalization. This result suggests that in problem solving, two constraints should be brought to bear in order to optimize generalization: lightening the cognitive load, but also deliberately encouraging the generalization process. Reducing the salience of important structural elements seems to be a means of facilitating the latter goal.

\section{REFERENCES}

Ahn, W., Brewer, W. F., \& Mooney, R. J. (1992). Schema acquisition from a single example. Journal of Experimental Psychology: Learning, Memory, \& Cognition, 18, 391-412.

Anolli, L., Antonietti, A., Crisafulli, L., \& Cantoia, M. (2001). Accessing source information in analogical problem-solving. Quarterly Journal of Experimental Psychology, 54A, 237-261.

BAssoK, M. (1990). Transfer of domain-specific problem-solving procedures. Journal of Experimental Psychology: Learning, Memory, \& Cognition, 16, 522-533.

BEVERIDGE, M., \& PARKINS, E. (1987). Visual representation in analogical problem solving. Memory \& Cognition, 15, 230-237.

Catrambone, R. (1995). Aiding subgoal learning: Effects on transfer. Journal of Educational Psychology, 87, 5-17.

CATRAMBONE, R. (1996). Generalizing solution procedures learned from examples. Journal of Experimental Psychology: Learning, Memory, \& Cognition, 22, 1020-1031.

Catrambone, R., \& HoLYOAK, K. J. (1989). Overcoming contextual limitation on problem-solving transfer. Journal of Experimental Psychology: Learning, Memory, \& Cognition, 15, 1147-1156.

Chandler, P., \& Sweller, J. (1991). Cognitive load theory and the format of instruction. Cognition \& Instruction, 8, 293-332.

Chandler, P., \& Sweller, J. (1992). The split-attention effect as a factor in the design of instruction. British Journal of Educational Psychology, 62, 233-246.

Chandler, P., \& Sweller, J. (1996). Cognitive load while learning to use a computer program. Applied Cognitive Psychology, 10, 1-20.

Chi, M. T. H., Feltovich, P. J., \& Glaser, R. (1981). Categorization and representation of physics problems by experts and novices. $\mathrm{Cog}$ nitive Science, 5, 121-152.

CLEMENT, C. A., \& GENTNER, D. (1991). Systematicity as a selection constraint in analogical mapping. Cognitive Science, 15, 89-132.
Cummins, D. D. (1992). Role of analogical reasoning in the induction of problem categories. Journal of Experimental Psychology: Learning, Memory, \& Cognition, 5, 1103-1124.

DeJong, G. F., \& Mooney, R. (1986). Explanation-based learning: An alternative view. Machine Learning, 1, 145-176.

DiDIERJEAN, A. (2003). Is case-based reasoning a source of knowledge generalization? European Journal of Cognitive Psychology, 15, 435453.

Elio, R., \& Anderson, J. R. (1981). The effects of category generalizations and instance similarity on schema abstraction. Journal of Experimental Psychology: Human Learning \& Memory, 7, 397-417.

FalKenhainer, B., Forbus, K. D., \& Gentner, D. (1989). The structuremapping engine: Algorithm and examples. Artificial Intelligence, 41, $1-63$.

Gentner, D. (1989). The mechanisms of analogical learning. In S. Vosniadou \& A. Ortony (Eds.), Similarity and analogical reasoning (pp. 199-241). New York: Cambridge University Press.

GenTner, D., \& Toupin, C. (1986). Systematicity and surface similarity in the development of analogy. Cognitive Science, 10, 277-300.

Gick, M. [L.], \& Holyoak, K. J. (1980). Analogical problem solving. Cognitive Psychology, 12, 306-355.

GiCK, M. [L.], \& HoLYOAK, K. J. (1983). Schema induction and analogical transfer. Cognitive Psychology, 15, 1-38.

GicK, M. L., \& MCGarrY, S. J. (1992). Learning from mistakes: Inducing analogous solution failures to a source problem produces later successes in analogical transfer. Journal of Experimental Psychology: Learning, Memory, \& Cognition, 18, 623-639.

Gick, M. L., \& PAterson, K. (1992). Do contrasting examples facilitate schema acquisition and analogical transfer? Canadian Journal of Psychology, 46, 539-550.

HEYDENBLUTH, C., \& HESSE, F. W. (1996). Impact of superficial similarity in the application phase of analogical problem solving. American Journal of Psychology, 109, 37-57.

HoLYOAK, K. J. (1984). Analogical thinking and human intelligence. In R. J. Sternberg (Ed.), Advances in the psychology of human intelligence (Vol. 2, pp. 199-230). Hillsdale, NJ: Erlbaum.

HOLYOAK, K. J., \& KoH, K. (1987). Surface and structural similarity in analogical transfer. Memory \& Cognition, 15, 332-340.

HolyOAK, K. J., \& THAGARD, P. (1989). Analogical mapping by constraint satisfaction. Cognitive Science, 13, 295-355.

Hummel, J. E., \& HoLYOAK, K. J. (1997). Distributed representations of structure: A theory of analogical access and mapping. Psychological Review, 104, 427-466.

KAPLAN, C. A., \& SimON, H. A. (1990). In search of insight. Cognitive Psychology, 22, 374-419.

KEANE, M. (1985). On drawing analogies when solving problems. British Journal of Psychology, 76, 449-458.

KEANE, M. (1987). On retrieving analogies when solving problems. Quarterly Journal of Experimental Psychology, 39A, 29-41.

KEANE, M. (1996). On adaptation in analogy: Tests of pragmatic importance and adaptability in analogical problem solving. Quarterly Journal of Experimental Psychology, 49A, 1062-1085.

Keane, M. T., Ledgeway, T., \& DufF, S. (1994). Constraints on analogical mapping: A comparison of three models. Cognitive Science, 18, 387-438.

KIERAS, D. E., \& BovaIR, S. (1986). The acquisition of procedures from text: A production system analysis of transfer of training. Journal of Memory \& Language, 25, 507-524.

MARMÈCHE, E., \& DIDIERJEAN, A. (2001). Is generalization conservative? European Journal of Cognitive Psychology, 13, 475-491.

Mooney, R. J. (1990). Learning plan schemata from observation: Explanation-based learning from plan recognition. Cognitive Science, 14, 483-509.

NeEDham, D. R., \& BegG, I. M. (1991). Problem-oriented training promotes spontaneous analogical transfer: Memory-oriented training promotes memory for training. Memory \& Cognition, 19, 543557.

Novick, L. R., \& HolyoaK, K. J. (1991). Mathematical problem solving by analogy. Journal of Experimental Psychology: Learning, Memory, \& Cognition, 17, 398-415.

PaAS, F. G. W. C., \& VAN MERRIËNBOER, J. J. G. (1994). Variability of worked examples and transfer of geometrical problem-solving skills: 
A cognitive-load approach. Journal of Educational Psychology, 86, 122-133.

PatAlano, A. L., \& Seifert, C. M. (1994). Memory for impasses during problem solving. Memory \& Cognition, 22, 234-242.

REED, S. K. (1987). A structure-mapping model for word problems. Journal of Experimental Psychology: Learning, Memory, \& Cognition, 13, 124-139.

REED, S. K. (1989). Constraints on the abstraction of solutions. Journal of Educational Psychology, 81, 532-540.

REED, S. K., \& BolstAD, C. A. (1991). Use of examples and procedures in problem solving. Journal of Experimental Psychology: Learning, Memory, \& Cognition, 17, 753-766.

ReEd, S. K., Dempster, A., \& EtTinger, M. (1985). Usefulness of analogous solutions for solving algebra word problems. Journal of Experimental Psychology: Learning, Memory, \& Cognition, 11, 106125.

ReEves, L. M., \& Weisberg, R. W. (1994). The role of content and abstract information in analogical transfer. Psychological Bulletin, $\mathbf{1 1 5}$, 381-400.

Ross, B. H. (1984). Remindings and their effects in learning a cognitive skill. Cognitive Psychology, 16, 371-416.

Ross, B. H. (1987). This is like that: The use of earlier problems and the separation of similarity effects. Journal of Experimental Psychology: Learning, Memory, \& Cognition, 13, 629-639.

Ross, B. H. (1989). Distinguishing types of superficial similarities: Different effects on the access and use of earlier problems. Journal of Experimental Psychology: Learning, Memory, \& Cognition, 15, 456468.

Ross, B. H., \& BRADShaw, G. L. (1994). Encoding effects of remindings. Memory \& Cognition, 22, 591-605.

Ross, B. H., \& KENNEDY, P. T. (1990). Generalizing from the use of ear- lier examples in problem solving. Journal of Experimental Psychology: Learning, Memory, \& Cognition, 16, 42-55.

SANDER, E., \& RichaRD, J. F. (1997). Analogical transfer as guided by an abstraction process: The case of learning by doing in text editing. Journal of Experimental Psychology: Learning, Memory, \& Cognition, 23, 1459-1483.

SCHANK, R. C. (1982). Dynamic memory, a theory of reminding and learning in computers and people. Cambridge: Cambridge University Press.

Schunn, C. D., \& Dunbar, K. (1996). Priming, analogy, and awareness in complex reasoning. Memory \& Cognition, 24, 271-284.

Sweller, J. (1988). Cognitive load during problem solving: Effects on learning. Cognitive Science, 12, 257-285.

Sweller, J. (1994). Cognitive load theory, learning difficulty, and instructional design. Learning \& Instruction, 4, 295-312.

Sweller, J. (1999). Instructional design in technical areas. Melbourne: ACER.

Sweller, J., Chandler, P., Tierney, P., \& Cooper, M. (1990). Cognitive load and selective attention as factors in the structuring of technical material. Journal of Experimental Psychology: General, 119, 176-192.

VanLehn, K. (1998). Analogy events: How examples are used during problem solving. Cognitive Science, 22, 347-388.

Wright, R. L., \& WhitTlesea, B. W. A. (1998). Implicit learning of complex structures: Active adaptation and selective processing in acquisition and application. Memory \& Cognition, 26, 402-420.

\section{NOTE}

1. For the sake of readability, we have given different names to the two Gick and McGarry (1992) problems used here. The problem we call "easy" corresponds to their "verbal-plus-diagram" problem, and our "difficult" problem corresponds to their "diagram-only" problem.

\section{APPENDIX A \\ The Partner Problem}

One Saturday night, at a local country dance, 40 people, 20 men and 20 women, showed up to dance. The dance was a "contra dance," in which men and women face each other in lines. From 8 to 10 p.m., there were 20 heterosexual couples (consisting of one man and one woman each; i.e., two men or two women cannot dance together) dancing on the floor. At 10 p.m., however, two women left, leaving 38 people to dance. Could the dance caller make arrangements so that the remaining people could all dance together at the same time in 19 heterosexual couples? The dance caller must remain a caller only, and cannot take a partner. Answer yes or no, and explain the reasons for your answer.

From "Learning from mistakes: Inducing analogous solution failures to a source problem produces later successes in analogical transfer," by M. L. Gick and S. J. McGarry, 1992, Journal of Experimental Psychology: Learning, Memory, \& Cognition, 18, p. 638. Copyright 1992 by the American Psychological Association. Reprinted with permission. 


\section{APPENDIX B \\ Difficult Version of the Dinner Party Problem}

Thirty-six people, 18 men and 18 women, are at a dinner party, as illustrated schematically below. The 36 people are seated at 18 tables. Each table only seats two people who are sitting right next to each other, either in a horizontal or vertical direction. If two people leave, as illustrated by the arrows in the diagram below, can 17 tables be arranged to seat the other 34 people? The people are not allowed to move. Explain your reasoning.

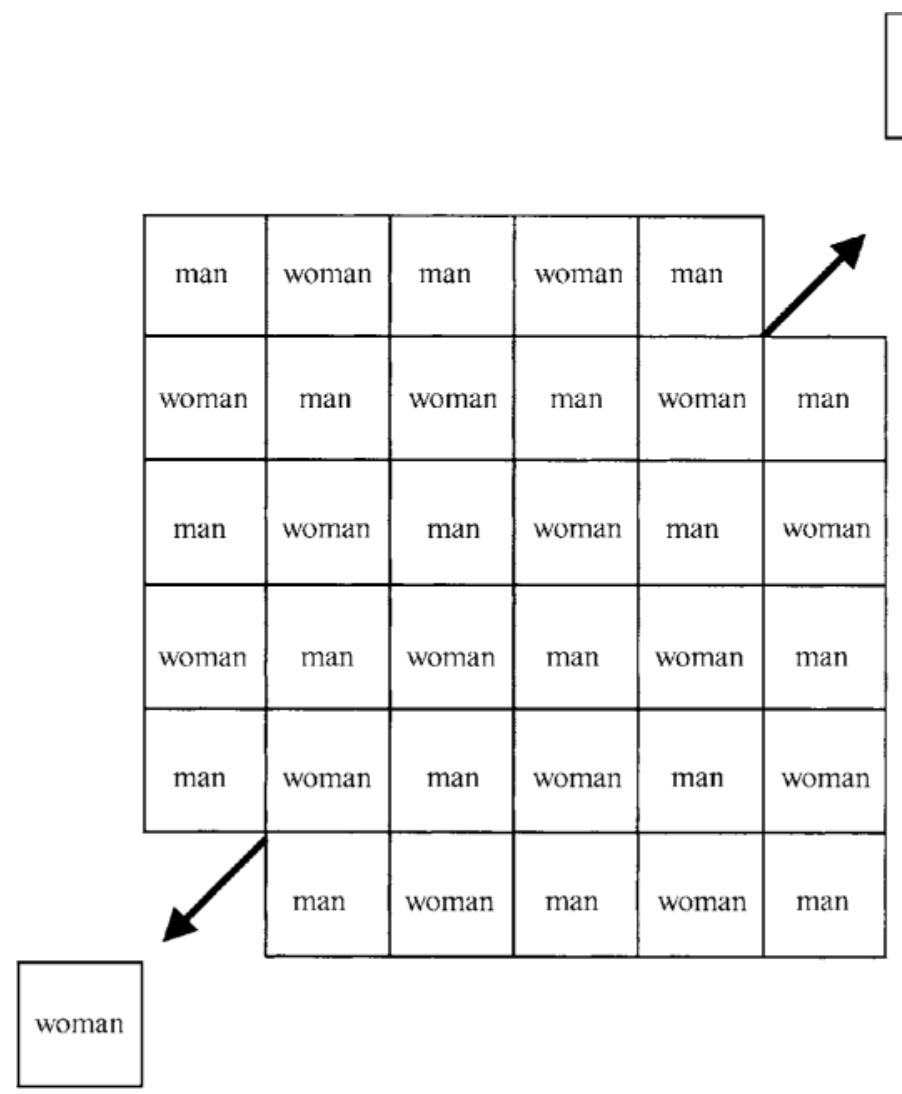

From "Learning from mistakes: Inducing analogous solution failures to a source problem produces later successes in analogical transfer," by M. L. Gick and S. J. McGarry, 1992, Journal of Experimental Psychology: Learning, Memory, \& Cognition, 18, p. 630. Copyright 1992 by the American Psychological Association. Reprinted with permission.

\section{APPENDIX C \\ Solution to the Dinner Party Problem}

It is important to notice that, since a table seats two people who are right next to each other, any given table must always have a man and a woman seated at it. In removing two women, there are two fewer women than men who are left. So, if a table must always seat a man and a woman, and there are fewer women than men, then the remaining 17 tables cannot seat the 34 people.

From "Learning from mistakes: Inducing analogous solution failures to a source problem produces later successes in analogical transfer," by M. L. Gick and S. J. McGarry, 1992, Journal of Experimental Psychology: Learning, Memory, \& Cognition, 18, p. 630. Copyright 1992 by the American Psychological Association. Reprinted with permission. 
APPENDIX D

Table D1

Subjects' Response Types on the Source and Target, by Group

\begin{tabular}{lccccr}
\hline & \multicolumn{5}{c}{ Target } \\
\cline { 2 - 5 } \multicolumn{1}{c}{ Source } & $\begin{array}{c}\text { Correct } \\
\text { Solution }\end{array}$ & $\begin{array}{c}\text { Nonparity } \\
\text { Failures }\end{array}$ & $\begin{array}{c}\text { One-Feature } \\
\text { Failures }\end{array}$ & $\begin{array}{c}\text { Other } \\
\text { Failures }\end{array}$ & $N$ \\
\hline Correct solution & \multicolumn{2}{c}{ Easy-Source Group } & & & \\
Nonparity failures & 4 & 5 & 2 & 2 & 13 \\
One-feature failures & 0 & 4 & 0 & 0 & 4 \\
Other failures & 2 & 3 & 1 & 0 & 6 \\
$N$ & 2 & 12 & 2 & 6 & 22 \\
& 8 & 24 & 5 & 8 & 45 \\
Correct solution & 1 & 1 & 0 & 0 & \\
Nonparity failures & 5 & 8 & 0 & 1 & 14 \\
One-feature failures & 1 & 4 & 0 & 0 & 5 \\
Other failures & 11 & 9 & 1 & 3 & 24 \\
$N$ & 18 & 22 & 1 & 4 & 45 \\
\hline
\end{tabular}

(Manuscript received January 30, 2003;

revision accepted for publication February 24, 2004.) 